\title{
Altered proteome of a Burkholderia pseudomallei mutant defective in short-chain dehydrogenase affects cell adhesion, biofilm formation and heat stress tolerance
}

\author{
Onrapak Reamtong ${ }^{1}$, Nitaya Indrawattana ${ }^{2}$, Amporn Rungruengkitkun ${ }^{2}$, Tipparat Thiangtrongjit ${ }^{1}$, Taksaon \\ Duangurai $^{2,3}$, Manas Chongsanguan ${ }^{2}$, Pornpan Pumirat ${ }^{\text {Corresp. } 2}$ \\ ${ }^{1}$ Department of Molecular Tropical Medicine and Genetics/Faculty of Tropical Medicine, Mahidol University, Bangkok, Thailand \\ 2 Department of Microbiology and Immunology/Faculty of Tropical Medicine, Mahidol University, Bangkok, Thailand \\ 3 Department of Companion Animal Clinical Sciences/Faculty of Veterinary Medicine, Kasetsart University, Bangkok, Thailand \\ Corresponding Author: Pornpan Pumirat \\ Email address: pornpan.pum@mahidol.ac.th
}

Burkholderia pseudomallei is a Gram-negative bacillus that causes melioidosis and is recognized as an important public health problem in southeast Asia and northeast Australia. The treatment of $B$. pseudomallei infection is hampered by resistance to a wide range of antimicrobial agents and no vaccine is currently available. At present, the underlying mechanisms of $B$. pseudomallei pathogenesis are poorly understood. In our previous study, we reported that a B. pseudomallei short-chain dehydrogenase (SDO; BPSS2242) mutant constructed by deletion mutagenesis showed reduced $B$. pseudomallei invasion and initial intracellular survival. This indicated that SDO is associated with the pathogenesis of melioidosis. In the present study, the role of $B$. pseudomallei SDO was further investigated using the SDO deletion mutant by a proteomic approach. The protein profiles of the SDO mutant and wild-type K96243 were investigated through gel-based proteomic analysis. Quantitative intensity analysis of three individual cultures of the $B$. pseudomallei SDO mutant revealed significant down-regulation of five protein spots compared with the wild-type. Q-TOF MS/MS identified the protein spots as a glutamate/aspartate $A B C$ transporter, prolyl-tRNA synthetase, Hsp70 family protein, quinone oxidoreductase and a putative carboxypeptidase. Functional assays were performed to investigate the role of these differentially expressed proteins in adhesion to host cells, biofilm induction and survival under heat stress conditions. The SDO deletion mutant showed a decreased ability to adhere to host cells. Moreover, biofilm formation and the survival rate of bacteria under heat stress conditions were also reduced in the mutant strain. Our findings provide insight into the role of SDO in the survival and pathogenesis of $B$. pseudomallei at the molecular level, which may be applied to the prevention and control of $B$. pseudomallei infection. 
$1 \quad$ Altered proteome of a Burkholderia pseudomallei mutant defective

2 in short-chain dehydrogenase affects cell adhesion, biofilm

formation and heat stress tolerance

\section{Onrapak Reamtong1, Nitaya Indrawattana², Amporn Rungruengkitkun², Tipparat \\ Thiangtrongjit ${ }^{1}$, Taksaon Duangurai ${ }^{2,3}$, Manas Chongsa-nguan², and Pornpan Pumirat ${ }^{2 *}$}

$7{ }^{1}$ Department of Molecular Tropical Medicine and Genetics, Faculty of Tropical Medicine,

8 Mahidol University, Bangkok, Thailand

92 Department of Microbiology and Immunology, Faculty of Tropical Medicine, Mahidol

10 University, Bangkok, Thailand

113 Department of Companion Animal Clinical Sciences, Faculty of Veterinary Medicine,

12 Kasetsart University, Bangkok, Thailand

14 *Corresponding author:

15 Email: pornpan.pum@mahidol.ac.th

\section{Abstract}

Burkholderia pseudomallei is a Gram-negative bacillus that causes melioidosis and is recognized as an important public health problem in southeast Asia and northeast Australia. The treatment of B. pseudomallei infection is hampered by resistance to a wide range of antimicrobial 
21 agents and no vaccine is currently available. At present, the underlying mechanisms of $B$.

22 pseudomallei pathogenesis are poorly understood. In our previous study, we reported that a $B$.

23 pseudomallei short-chain dehydrogenase (SDO; BPSS2242) mutant constructed by deletion

24 mutagenesis showed reduced B. pseudomallei invasion and initial intracellular survival. This

25 indicated that SDO is associated with the pathogenesis of melioidosis. In the present study, the

26 role of $B$. pseudomallei SDO was further investigated using the SDO deletion mutant by a

27 proteomic approach. The protein profiles of the SDO mutant and wild-type K96243 were

28 investigated through gel-based proteomic analysis. Quantitative intensity analysis of three

29 individual cultures of the B. pseudomallei SDO mutant revealed significant down-regulation of

30 five protein spots compared with the wild-type. Q-TOF MS/MS identified the protein spots as a

31 glutamate/aspartate $\mathrm{ABC}$ transporter, prolyl-tRNA synthetase, Hsp70 family protein, quinone

32 oxidoreductase and a putative carboxypeptidase. Functional assays were performed to investigate

33 the role of these differentially expressed proteins in adhesion to host cells, biofilm induction and

34 survival under heat stress conditions. The SDO deletion mutant showed a decreased ability to

35 adhere to host cells. Moreover, biofilm formation and the survival rate of bacteria under heat

36 stress conditions were also reduced in the mutant strain. Our findings provide insight into the

37 role of SDO in the survival and pathogenesis of B. pseudomallei at the molecular level, which

38 may be applied to the prevention and control of B. pseudomallei infection.

40 Keywords: Burkholderia pseudomallei, short-chain dehydrogenase, proteome, adhesion, biofilm

41 formation, heat stress 


\section{Introduction}

Burkholderia pseudomallei is the etiological agent of melioidosis in both humans and

animals. It is a natural inhabitant of soil, stagnant water and rice paddies, where the disease is endemic. Melioidosis endemic areas include South-East Asia, in particular northeastern Thailand and northern Australia (Wuthiekanun et al., 1995; Cheng and Currie, 2005). Rice farmers are considered to be at high risk of exposure during the monsoonal and rainy season (Chaowagul et al., 1989; Cheng and Currie, 2005; Inglis and Sagripanti, 2006; Wiersinga et al., 2006), particularly when planting and harvesting in mud and surface water in rice fields. Infection occurs by inoculation through skin abrasions or inhalation, and ranges from acute to chronic. Acute infection often involves septicemia, resulting in death within days of exposure. The longest reported incubation period between initial acquisition and subsequent infection is a remarkable 62 years. Furthermore, a high rate of relapse has been recognized (Ngauy et al., 2005). B. pseudomallei exhibits resistance to diverse groups of antibiotics including thirdgeneration cephalosporins, penicillins, rifamycins, macrolides, quinolones and aminoglycosides (Cheng and Currie, 2005). The antibiotic ceftazidime is the drug of choice for treatment, either alone or in combination with other antibiotics such as chloramphenicol, doxycycline and trimethoprim-sulfa methoxazole. Currently, there is no vaccine available against melioidosis. Owing to its aerosol infectivity, the severe course of infection, and the absence of vaccines and fully effective treatments, B. pseudomallei is classified as a hazard category three pathogen and considered a potential biothreat agent (Cheng and Currie, 2005; Cheng et al., 2005).

B. pseudomallei can survive as a free-living organism in environmental niches, such as soil and water; as well as being parasitic to living organisms such as amoeba, plants, fungi and animals (Inglis and Sagripanti, 2006). Furthermore, this bacterium is able to live and multiply 
66 under various adverse conditions (Choy et al., 2000; Dance, 2000). It is likely that this organism

67 has developed strategies to survive in both the natural environment and in its respective hosts. In

68 the human host, B. pseudomallei can infect both phagocytic and non-phagocytic cells. Once the

69 bacteria adheres to the target receptor, it invades the host cell cytoplasm. Following

70 internalization, $B$. pseudomallei has evolved mechanisms to enter and escape from phagosomes,

71 passing into the host cell cytosol (Wiersinga et al., 2006). In addition, B. pseudomallei induces

72 actin polymerization that leads to bacterial motility and the formation of host-cell-membrane

73 protrusions tipped by intracellular bacteria that project into adjacent cells (Stevens et al., 2005).

74 These protrusions are believed to underlie the ability of $B$. pseudomallei to uniquely promote

75 multinucleated giant cell formation (Kespichayawattana et al., 2000). This characteristic

76 phenotype has been observed in the tissues of melioidosis patients (Wong et al., 1995). However,

77 the molecular mechanisms behind this pathogen's survival and pathogenesis remain largely

78 unknown.

79 The short-chain dehydrogenase/oxidoreductase (SDO) is an enzyme that

80 catalyzes reversible $\mathrm{NAD}(\mathrm{P})(\mathrm{H})$-dependent reactions (Kallberg et al., 2010). In the

81 dehydrogenase reaction, a hydride (i.e., a proton plus two electrons) is removed from the

82 substrate and transferred to an electron acceptor, which depending on the enzyme is

$83 \mathrm{NAD}^{+}$or $\mathrm{NADP}^{+}$. The role of SDO in bacterial pathogenesis has been described in Pseudomonas

84 aeruginosa. $P$. aeruginosa SDO is involved in reduced pyocyanin production, decreased

85 motility, poor biofilm formation and absent paralysis of Caenorhabditis elegans (Bijtenhoorn et

86 al., 2011). Recently, SDO-defective B. pseudomallei showed a significant reduction in glucose

87 dehydrogenase (GDH) activity, invasion and survival in host cells (Pumirat et al., 2014). 
88 However, the underlying mechanism of SDO in the pathogenesis of this bacterium remained

89 unknown.

90 In the present study, we explored the roles of SDO in the pathogenesis and survival of $B$.

91 pseudomallei. We performed proteomic analysis to investigate SDO-associated proteins in $B$.

92 pseudomallei. The cellular proteome of K96243 wild-type was compared with that of the SDO

93 mutant using a gel-based approach. The phenotypes associated with the differentially expressed

94 proteins were studied in the wild-type and mutant strains, and included adherence to host cells,

95 biofilm formation and survival under heat stress. Taken together, our data provide insight into

96 the molecular mechanisms of SDO in B. pseudomallei pathogenesis and survival.

\section{Materials and Methods}

99 Bacterial strains, cell lines and culture conditions

100 B. pseudomallei wild-type K96243, the SDO mutant (Pumirat et al., 2014) and the 101 complemented strain (Pumirat et al., 2014) were cultured in Luria-Bertani (LB) medium 102 (Difco $^{\mathrm{TM}}$, Becton Dickinson, USA) and grown at $37^{\circ} \mathrm{C}$. To determine the growth kinetics of $B$. 103 pseudomallei, the overnight culture of $B$. pseudomallei adjusted to the optical density at $600 \mathrm{~nm}$ $104\left(\mathrm{OD}_{600}\right) 0.5$ was inoculated 1:500 into standard LB broth. Every $2 \mathrm{~h}$ after being inoculated, the 105 optical density of cultures at various time points was recorded.

Cell lines used in this study included A549 (human respiratory epithelial cells) and HFF-

1071 (human skin fibroblast), which were obtained from the American Type Culture Collection 108 (ATCC, Manassas, VA, USA). The A549 cell line was maintained in Ham's F-12 medium 109 supplemented with $10 \%(\mathrm{v} / \mathrm{v})$ heat-inactivated fetal bovine serum (FBS), while the HFF-1 skin

110 fibroblast cell line was maintained in Dulbecco's modified Eagle's medium (DMEM) 
111 supplemented with $10 \%(\mathrm{v} / \mathrm{v})$ heat-inactivated FBS. All cells were cultured in a $5 \% \mathrm{CO}_{2}$

112 atmosphere at $37^{\circ} \mathrm{C}$ in a humidified incubator.

113

114 Protein lysate preparation

115 B. pseudomallei wild-type (K96243) and SDO mutant cells were resuspended in lysis

116 buffer containing of $8 \mathrm{M}$ urea (OmniPur ${ }^{\circledR}$, Germany), $2 \mathrm{M}$ thiourea (Merck, Germany), 4\%

117 CHAPS (Thermo Scientific, USA) and $50 \mathrm{mM}$ dithiothreitol (DTT) (OmniPur®). The lysates

118 were ultrasonicated on ice and the supernatants were collected after centrifugation at $12,000 \times \mathrm{g}$

119 for $5 \mathrm{~min}$ at $4^{\circ} \mathrm{C}$. A 2-D clean-up kit (GE Healthcare, Germany) and Quick Start Bradford

120 protein assay (Bio-Rad, USA) were used for protein precipitation and quantification.

121

\section{Two-dimensional gel electrophoresis (2-DE)}

123 Proteins were rehydrated on a 7-cm immobilized $\mathrm{pH}$ gradient (IPG) strip (pH 3-10, NL)

124 (GE Healthcare) overnight in $5 \mathrm{M}$ urea, $2 \mathrm{M}$ thiourea, $50 \mathrm{mM}$ DTT, 4\% CHAPS and IPG buffer.

125 After isoelectric focusing by an Ettan ${ }^{\mathrm{TM}}$ IPGphor $^{\mathrm{TM}} 3$ (GE Healthcare), proteins were reduced in

$12650 \mathrm{mM}$ DTT for $15 \mathrm{~min}$ and alkylated in $125 \mathrm{mM}$ iodoacetamide (IAA) in $6 \mathrm{M}$ urea, $75 \mathrm{mM}$

127 Tris- $\mathrm{HCl}, 70 \mathrm{mM}$ SDS and 30\% glycerol for $15 \mathrm{~min}$. The samples were further separated by $12 \%$

128 acrylamide gel (Bio-Rad). Following 2-DE, all gels were stained with Coomassie blue. Three

129 biological replicates were performed for each sample. The spot volume was used for

130 quantification. Spots of interest that showed at least a two-fold difference and an ANOVA P

131 value $\leq 0.05$ were excised for protein identification. 


\section{In-gel tryptic digestion}

134 Each gel piece was destained with $50 \%$ acetonitrile (ACN) in $50 \mathrm{mM}$ ammonium

135 bicarbonate (Merck, USA). Proteins in gel spots were reduced and alkylated by 4 mM DTT and 136250 mM IAA, respectively. The samples were dehydrated with $100 \%$ ACN (Thermo Scientific, 137 USA). Trypsin (Sigma-Aldrich, USA, T6567) was added for digestion overnight at $37^{\circ} \mathrm{C}$. After 138 extraction with $100 \% \mathrm{ACN}$, peptides were stored at $-20^{\circ} \mathrm{C}$.

Mass spectrometry analysis

An Ultimate ${ }^{\circledR} 3000$ Nano-LC system (Thermo Scientific) was used for peptide separation. A microTOF-Q II (Bruker, Germany) was used to analyze MS and MS/MS spectra at $\mathrm{m} / \mathrm{z}$ 400-2000 and m/z 50-1500, respectively. The acquisition was controlled by HyStar ${ }^{\mathrm{TM}}$ version 3.2 (Bruker). DataAnalysis ${ }^{\mathrm{TM}}$ software version 3.4 (Bruker) was used to convert raw data format (.d) files to mascot generic files (.mgf), which were further searched by Mascot software (Matrix Science, USA). A SwissProt bacterial database was set for protein identification.

\section{RNA preparation and real-time RT-PCR}

RNA was isolated from stationary phase growth of B. pseudomallei cells grown at $37^{\circ} \mathrm{C}$

by adding $10 \mathrm{ml}$ of RNAprotect bacterial reagent (Qiagen) to $5 \mathrm{ml}$ of bacterial culture and

151 incubating for $5 \mathrm{~min}$ at room temperature. Subsequently, total RNA was extracted from the

152 bacterial pellets using Trizol (Invitrogen, Carlsbad, CA, USA) according to the manufacturer's

153 instructions and was treated with DNase (NEB, MA, USA) for $10 \mathrm{~min}$ at $37^{\circ} \mathrm{C}$ before use. 
154 Standard PCR for the 23S RNA gene was used to verify that there was no gDNA contamination

155 in the DNase-treated RNA samples. Real time RT-PCR was performed using the Brilliant II

156 SYBR ${ }^{\circledR}$ Green QPCR Master Mix, one step (Agilent Technologies, Santa Clara, CA, USA).

157 Amplifications of five genes (kex, wzt, qor, proS and $h s p$ ) were performed under the following

158 conditions: reverse transcription at $50^{\circ} \mathrm{C}$ for $30 \mathrm{~min}$, enzyme activation at $95^{\circ} \mathrm{C}$ for $10 \mathrm{~min}$, then

15940 cycles of denaturation at $95^{\circ} \mathrm{C}$ for $30 \mathrm{~s}$, annealing at $55^{\circ} \mathrm{C}$ for $1 \mathrm{~min}$, and melting curve

160 analysis at $72^{\circ} \mathrm{C}$ for $1 \mathrm{~min}$ in a CFX96 Touch $^{\mathrm{TM}}$ Real-Time PCR Detection System (Bio-Rad,

161 Singapore) as previously described (Pumirat et al., 2017). The primer sequences are shown in

162 Table 1. Relative mRNA levels were determined by fold-changes in expression and calculated by

$1632^{-\Delta \Delta C T}$ using the relative mRNA levels of $23 \mathrm{~S}$ RNA, and the expression of a representative

164 house-keeping gene as a baseline for comparison.

165

166 Heat resistance assay

167 A heat stress resistance assay was performed as described previously (Pumirat et al., 168 2017) with some modifications. Briefly, B. pseudomallei cultured in LB medium at $37^{\circ} \mathrm{C}$ for $6 \mathrm{~h}$ 169 were washed with phosphate-buffered saline (PBS) and resuspended in PBS to an OD $_{600}$ of 0.15.

170 One milliliter of the bacterial suspension was then added into a prewarmed tube and incubated at $17150^{\circ} \mathrm{C}$ for $15 \mathrm{~min}$. Before and after heat challenge, bacterial survival was enumerated on LB agar 172 plates after incubating at $37^{\circ} \mathrm{C}$ for $24 \mathrm{~h}$. The number of surviving bacteria was expressed as a 173 percentage of the viable cells. \% Survival $=$ CFU (heat exposure) $\times 100 / C F U$ (without heat 174 exposure)

175

176 Biofilm formation assay 
178 as previously described (Pumirat et al., 2017). Each B. pseudomallei strain was assayed with at

179 least eight replicates per experiment, along with positive and negative controls. The optical

180 density was measured at $630 \mathrm{~nm}$ using a microplate reader (Bio-Rad). The biofilm formation

181 capacity was calculated as the $\mathrm{OD}_{630}$ of the test strain divided by the $\mathrm{OD}_{630}$ of the negative

182 control.

183

184 Adhesion assay

185 The adhesion of B. pseudomallei with human respiratory and skin cell lines was studied 186 as previously described (Essex-Lopresti et al., 2005). Bacterial inocula were prepared from

187 overnight cultures grown in LB broth, incubated statically for $18 \mathrm{~h}$ at $37^{\circ} \mathrm{C}$. Monolayers were

188 infected with diluted bacterial cultures at a multiplicity of infection (MOI) of 100 for $1 \mathrm{~h}$ at $37^{\circ} \mathrm{C}$.

189 Nonadherent bacteria were removed by five washes with PBS. Monolayers were lysed with $0.1 \%$

190 (vol/vol) Triton X-100 for $30 \mathrm{~min}$ at $37^{\circ} \mathrm{C}$, and adherent cell-associated bacteria were

191 enumerated by plate counts.

192

193 Statistical analysis

194 All assays were conducted at least in triplicate and statistical analyses of independent 195 experiments were performed by ANOVA with a 5\% confidence interval in GraphPad Prism 5 196 program (Statcon). Results were considered significant at a $\mathrm{P}$ value of $<0.05$. 


\section{Results}

198 Altered proteome in the $B$. pseudomallei SDO mutant

As SDO activity facilitates $B$. pseudomallei invasion and affects the initiation of

200

201

202

203

204

205

206

207

208

successful intracellular infection, we predicted that the expression of several proteins may be modulated by SDO. To investigate the molecular mechanisms of SDO, we performed proteomic analysis of the SDO mutant compared with the K96243 wild-type. Proteins extracted from wildtype and mutant bacteria were resolved in 2-D gels, individually ( $n=3$ gels for each group; total $n=6$ gels). Figure 1 shows representative 2-D gels of cellular proteins extracted from the K96243 wild-type and SDO mutant. Up to 497 protein spots were visualized by Coomassie blue staining. Among these, quantitative intensity analysis and statistics revealed five differentially expressed protein spots (with $\mathrm{P}<0.05$ ) between the K96243 wild-type and SDO mutant strains. These differentially expressed proteins were subsequently identified by LC-MS/MS analysis. The protein identification results, as well as the quantitative data, are shown in Table 2 and Supplement Information 1-5. The five differentially expressed proteins were identified as a glutamate/aspartate $\mathrm{ABC}$ transporter, prolyl-tRNA synthetase, Hsp70 family protein, quinone oxidoreductase and putative carboxypeptidase.

To confirm the level of gene expression, these five proteins were quantified by qRTPCR. In comparison to the wild-type strain, the expression levels of these five genes were obviously decreased in the SDO mutant (Figure 2), which was consistent with the proteomic findings. The SDO complement strain was able to recover the expression levels of these five genes (Figure 2). 
219 strain was determined to exclude the possibility that protein and RNA expression levels may

220 result from the difference of bacterial growth. No significant difference in growth among $B$.

221 pseudomallei strains (Supplement Information 6).

223 Reduced adhesion of the $B$. pseudomallei SDO mutant to host cells Hsp70 family proteins were down-regulated in the SDO mutant. These two proteins have been reported to play a role in bacterial adhesion (Leon-Kempis Mdel et al., 2006; Ghazaei, 2017). Adhesion is important for bacterial survival and the spread of bacterial cells, and is therefore a phenotype associated with virulence. $B$. pseudomallei is a facultative, intracellular bacteria that is able to adhere to, and invade, host cells (Kespichayawattana et al., 2000). Hence, we investigated the involvement of SDO in B. pseudomallei adhesion. B. pseudomallei wild-type and the SDO mutant were examined for their ability to adhere to A549 human respiratory and HFF-1 human skin fibroblast cell lines; since infection with this bacterium occurs by inoculation through inhalation and skin abrasions. As shown in Figure 3A, the B. pseudomallei SDO mutant showed a significantly lower level of adherence compared with the K96243 wild-type to the A549 cell 235 line $(\mathrm{P}=0.0026)$ and to the HFF-1 cell line $(\mathrm{P}=0.0205)$. The SDO complemented strain recovered the adhesion of the SDO mutant to a similar level to that of the wild-type. These data 237 suggested the role of SDO in the in vitro adherence of $B$. pseudomallei to the A549 cell line. 
239 Decreased biofilm formation in the $B$. pseudomallei SDO mutant

240 Down-regulation of the Hsp70 family protein in the SDO mutant has been reported to be

241 involved in biofilm formation (Arita-Morioka et al., 2015). The ability of bacteria to form

242 biofilm is crucial for their survival in adverse environments. Some dehydrogenases are necessary

243 for biofilm formation by bacteria (Bijtenhoorn et al., 2011). To investigate the role of SDO in

244 biofilm formation, the abilities of B. pseudomallei wild-type and the SDO mutant to induce

245 biofilm were evaluated (Figure 3B). The SDO mutant showed a significantly reduced ability to

246 induce biofilm compared with the wild-type strain $(\mathrm{P}=0.0065)$, suggesting that SDO plays a

247 role in the biofilm formation of $B$. pseudomallei.

Impaired heat stress tolerance of the B. pseudomallei SDO mutant

Many studies have reported that dehydrogenases are associated with protection of

bacterial cells against environmental stress (Fu et al., 1989; Cabiscol et al., 2000; Liu et al., 2001; Messner and Imlay, 2002; Hoper et al., 2005; Weerakoon et al., 2009; Miller et al., 2010).

We also found that the SDO mutant down-regulated the expression of Hsp70 family protein and quinone oxidoreductase, which plays a role in heat stress tolerance (Liu et al., 2008; Ghazaei, 2017). Thus, the effect of SDO on heat resistance in B. pseudomallei was evaluated. $B$. pseudomallei wild-type and the SDO mutant were cultured in LB broth, followed by heating at $50^{\circ} \mathrm{C}$ for 15 min. As shown in Figure $3 \mathrm{C}$, a significant difference in heat resistance was detected between $B$. pseudomallei wild-type and the SDO mutant $(\mathrm{P}=0.0343)$. The mean and standard deviation (SD) of bacterial survival in medium containing $150 \mathrm{mM}$ of B. pseudomallei wild-type 
261 SDO mutant were $8.2 \pm 1.0 \%$. These data clearly revealed that SDO was associated with the

262 resistance of $B$. pseudomallei to heat stress.

263

264 Discussion

265 B. pseudomallei is a soil saprophyte and the causative agent of melioidosis, a disease 266 endemic in southeast Asia and northern Australia (Wuthiekanun et al., 1995). This bacterium can

267 survive for prolonged periods under various environmental conditions and in various hosts. $B$.

268 pseudomallei possesses a variety of bacterial factors/enzymes to facilitate its survival and 269 pathogenesis. Among these, SDO is an enzyme that plays a role in the pathogenesis of several 270 bacteria including P. aeruginosa (Bijtenhoorn et al., 2011) and B. pseudomallei (Pumirat et al., 271 2014). In B. pseudomallei, SDO is essential for the processes of invasion and intracellular 272 replication (Pumirat et al., 2014). This suggests that SDO activity may modulate the expression 273 of several essential proteins to facilitate the successful infection of B. pseudomallei.

274 To obtain an overall view of the SDO-modulated response of $B$. pseudomallei, we 275 performed a comparative proteomic analysis using the 2-D-gel technique and mass spectrometry. 276 Interestingly, five differentially expressed proteins were identified, namely, a glutamate/aspartate 277 ABC transporter, prolyl-tRNA synthetase, Hsp70 family protein, quinone oxidoreductase and a 278 putative carboxypeptidase. All of these proteins were down-regulated (SDO mutant/K96243 279 wild-type ratios ranged from 0.00 to 0.23 ; average $=0.10$ ). Although only a limited set of 280 differentially expressed proteins could be detected between the SDO mutant and K96243 wild281 type, these proteins were able to provide a starting point for more detailed analysis of SDO282 modulated bacterial pathways. 
In general, the bacterial response to environmental stress is orchestrated by the 284 expression of a family of proteins termed the heat shock proteins, which include Hsp70 family 285 protein. Hsp70 protein is essential for bacterial growth under various stress conditions including 286 high temperature (Ghazaei, 2017). Heat shock proteins play a significant role in maintaining 287 correct protein configurations to avoid cellular damage. Furthermore, Hsp70 protein plays a role 288 in pathogenesis (Ghazaei, 2017) and biofilm formation (Arita-Morioka et al., 2015). During 289 infection, bacteria activate their heat shock genes to protect their cellular machinery from host defense mechanisms, thereby enhancing their virulence. Therefore, down-regulation of Hsp70 family protein in the SDO mutant was concordant with the phenotype of the SDO mutant, which showed impaired heat stress tolerance, biofilm formation and adhesion ability.

294 homologs from the C-terminal. The activity of this enzyme has been detected in a number of 295 clinical pathogens including Acinetobacter baumannii, Campylobacter jejuni, Listeria monocytogenes, Pseudomonas aeruginosa and Streptococcus agalactiae (Lough et al., 2016). A report showed that the hydrolysis of peptidoglycan LD-carboxypeptidase Pgp2 influences the helical cell shape and pathogenic properties of $C$. jejuni (Frirdich et al., 2014). Thus, it is possible that carboxypeptidase plays a synergistic role with SDO in the hydrolysis of the protein substrates for B. pseudomallei infection. import and export of a variety of molecules across cell membranes (Holland and Blight, 1999).

303 The significance of the glutamate/aspartate ABC transporter in bacterial pathogenesis has been 304 reported in $C$. jejuni. The PEB1 aspartate/glutamate $\mathrm{ABC}$ transporter serves as an adhesin for $C$. 305 jejuni adhesion (Leon-Kempis Mdel et al., 2006). Here, expression of the glutamate/aspartate 
$306 \mathrm{ABC}$ transporter protein was found to be reduced in the SDO mutant, leading to defective

307 adhesion. This suggested that the glutamate/aspartate $\mathrm{ABC}$ transporter protein was involved in

308 adhesion of the B. pseudomallei SDO mutant.

Quinone oxidoreductase is a multisubunit integral membrane enzyme that operates in the

310 respiratory chains of both bacteria and eukaryotic organelles (Spero et al., 2015). Although there

311 is no direct evidence demonstrating that quinone oxidoreductase is essential for B. pseudomallei

312 survival under adverse conditions, several reports have demonstrated such a role in other bacteria

313 (Liu et al., 2008; Ryan et al., 2014). For example, in Escherichia coli, significantly higher

314 survival rates were observed in E. coli strain YB overexpressing quinone oxidoreductase than in

315 the control strain when treated with heat shock and oxidative stressors such as $\mathrm{H}_{2} \mathrm{O}_{2}$ and

316 menadione (Liu et al., 2008). This supported the hypothesis that quinone oxidoreductase is

317 important for bacterial survival under stress conditions. In our study, we suggested that the

318 down-regulation of quinone oxidoreductase together with the down-regulation of stress response

319 proteins, as mentioned above, most likely led to the decreased survival of the SDO mutant under

320 heat stress conditions.

321 As in other organisms, during translation in bacterial cells each amino acid is carried by a

322 specific tRNA to the translation site. Prolyl-tRNA synthetase is one of aminoacyl-tRNA

323 synthetases that catalyzes the condensation of a specific amino acid to its cognate tRNA (Crepin

324 et al., 2006). This enzyme is therefore required for bacterial translation, which is a key cellular

325 process. There is no previously reported evidence indicating the role of this enzyme in bacteria

326 survival and virulence, with only one report demonstrating that cysteinyl and lysyl-tRNA

327 synthetases are essential for the growth of Mycobacterium smegmatis (Ravishankar et al., 2016). 
328 Further examination of the role of prolyl-tRNA synthetase and its association with SDO is

329 required to further our understanding of their functional significance in B. pseudomallei.

330

331 Conclusions

332 Proteomic analysis revealed a set of B. pseudomallei cellular proteins that were altered in

333 the SDO mutant compared with the wild-type strain. Down-regulation of a set of differentially

334 expressed proteins was detected in the mutant, offering insight into the role of SDO in heat stress

335 tolerance and biofilm formation in B. pseudomallei. In addition, in vitro studies of infected host

336 cells indicated that SDO was also involved in the adhesion of B. pseudomallei to human host

337 cells such as lung epithelial and skin fibroblast cells. The findings of this study provide further

338 insight into the roles and functions of B. pseudomallei SDO, which may be beneficial in the

339 development of prevention and control strategies.

340

341 Competing interests

342 The authors declare that the research was conducted in the absence of any commercial or

343 financial relationships that could be construed as a potential conflict of interest. 


\section{Authors' contributions}

PP designed the study. OR and TT performed proteomic analysis. NI and TD carried out real-time RT-PCR experiments. AR performed the biofilm formation and heat stress assays. PP carried out adhesion examinations. PP and OR analyzed the data, and wrote the manuscript. PP and MC contributed reagents/materials/analysis tools. PP approved the final draft. All authors read and approved the final version of the manuscript.

\section{Acknowledgments}

This work was supported by a research grant by Mahidol University, Fiscal year 2016 and 2018.

\section{References}

357

Arita-Morioka, K., Yamanaka, K., Mizunoe, Y., Ogura, T., and Sugimoto, S. (2015). Novel strategy for biofilm inhibition by using small molecules targeting molecular chaperone DnaK. Antimicrob Agents Chemother 59(1), 633-641. doi: 10.1128/AAC.04465-14

Bijtenhoorn, P., Mayerhofer, H., Muller-Dieckmann, J., Utpatel, C., Schipper, C., Hornung, C., Szesny, M., Grond, S., Thurmer, A., Brzuszkiewicz, E., Daniel, R., Dierking, K., Schulenburg, H., and Streit, W.R. (2011). A novel metagenomic short-chain dehydrogenase/reductase attenuates Pseudomonas aeruginosa biofilm formation and virulence on Caenorhabditis elegans. PLoS One 6(10), e26278. doi: 10.1371/journal.pone.0026278

Cabiscol, E., Tamarit, J., and Ros, J. (2000). Oxidative stress in bacteria and protein damage by reactive oxygen species. Int Microbiol 3(1), 3-8. 
368 Chaowagul, W., White, N.J., Dance, D.A., Wattanagoon, Y., Naigowit, P., Davis, T.M., 369 Looareesuwan, S., Pitakwatchara, N. (1989). Melioidosis: a major cause of community$370 \quad$ acquired septicemia in northeastern Thailand. J Infect Dis 159(5), 890-899.

371 Cheng, A.C., and Currie, B.J. (2005). Melioidosis: epidemiology, pathophysiology, and 372 management. Clin Microbiol Rev 18(2), 383-416.

373

Cheng, A.C., Dance, D.A., and Currie, B.J. (2005). Bioterrorism, Glanders and melioidosis. Euro Surveill 10(3), E1-2; author reply E1-2.

Choy, J.L., Mayo, M., Janmaat, A., and Currie, B.J. (2000). Animal melioidosis in Australia. Acta Trop 74(2-3), 153-158.

Crepin, T., Yaremchuk, A., Tukalo, M., and Cusack, S. (2006). Structures of two bacterial prolyl-tRNA synthetases with and without a cis-editing domain. Structure 14(10), 15111525. doi: 10.1016/j.str.2006.08.007.

Dance, D.A. (2000). Ecology of Burkholderia pseudomallei and the interactions between environmental Burkholderia spp. and human-animal hosts. Acta Trop 74(2-3), 159-168.

Essex-Lopresti, A.E., Boddey, J.A., Thomas, R., Smith, M.P., Hartley, M.G., Atkins, T., Brown, N.F., Tsang, C.H., Peak, I.R., Hill, J., Beacham, I.R., and Titball, R.W. (2005). A type IV pilin, PilA, contributes to adherence of Burkholderia pseudomallei and virulence in vivo. Infect Immun 73(2), 1260-1264. doi: 10.1128/IAI.73.2.1260-1264.2005.

Frirdich, E., Vermeulen, J., Biboy, J., Soares, F., Taveirne, M.E., Johnson, J.G., DiRita, V.J., Girardin, S.E., Vollmer, W., and Gaynor, E.C. (2014). Peptidoglycan LDcarboxypeptidase Pgp2 influences Campylobacter jejuni helical cell shape and pathogenic properties and provides the substrate for the DL-carboxypeptidase Pgp1. $J$ Biol Chem 289(12), 8007-8018. doi: 10.1074/jbc.M113.491829 
391 Fu, H.S., Hassett, D.J., and Cohen, M.S. (1989). Oxidant stress in Neisseria gonorrhoeae:

392 adaptation and effects on L-(+)-lactate dehydrogenase activity. Infect Immun 57(7), $393 \quad 2173-2178$.

394 Ghazaei, C. (2017). Role and mechanism of the Hsp70 molecular chaperone machines in 395 bacterial pathogens. J Med Microbiol 66(3), 259-265. doi: 10.1099/jmm.0.000429.

396 397 398

Holland, I.B., and Blight, M.A. (1999). ABC-ATPases, adaptable energy generators fuelling transmembrane movement of a variety of molecules in organisms from bacteria to humans. J Mol Biol 293(2), 381-399. doi: 10.1006/jmbi.1999.2993

Hoper, D., Volker, U., and Hecker, M. (2005). Comprehensive characterization of the contribution of individual SigB-dependent general stress genes to stress resistance of Bacillus subtilis. J Bacteriol 187(8), 2810-2826. doi:10.1128/JB.187.8.2810-2826.2005.

Inglis, T.J., and Sagripanti, J.L. (2006). Environmental factors that affect the survival and persistence of Burkholderia pseudomallei. Appl Environ Microbiol 72(11), 6865-6875. doi: 10.1128/AEM.01036-06.

Kallberg, Y., Oppermann, U., and Persson, B. (2010). Classification of the short-chain dehydrogenase/reductase superfamily using hidden Markov models. FEBS J 277(10), 2375-2386. doi: 10.1111/j.1742-4658.2010.07656.x

Kespichayawattana, W., Rattanachetkul, S., Wanun, T., Utaisincharoen, P., and Sirisinha, S. (2000). Burkholderia pseudomallei induces cell fusion and actin-associated membrane protrusion: a possible mechanism for cell-to-cell spreading. Infect Immun 68(9), 53775384.

Leon-Kempis Mdel, R., Guccione, E., Mulholland, F., Williamson, M.P., and Kelly, D.J. (2006). The Campylobacter jejuni PEB1a adhesin is an aspartate/glutamate-binding protein of an 
414

415

416

417

418

419

420

421

422

423

424

425

426

427

428

429

430

431

432

433

434

435

436

ABC transporter essential for microaerobic growth on dicarboxylic amino acids. Mol Microbiol 60(5), 1262-1275. doi: 10.1111/j.1365-2958.2006.05168.x.

Liu, G., Zhou, J., Jin, R., Zhou, M., Wang, J., Lu, H., and Qu, Y. (2008). Enhancing survival of Escherichia coli by expression of azoreductase AZR possessing quinone reductase activity. Appl Microbiol Biotechnol 80(3), 409-416. doi: 10.1007/s00253-008-1555-6.

Liu, L., Hausladen, A., Zeng, M., Que, L., Heitman, J., Stamler, J.S., and Steverding, D. (2001). Nitrosative stress: protection by glutathione-dependent formaldehyde dehydrogenase. Redox Rep 6(4), 209-210.

Lough, F., Perry, J.D., Stanforth, S.P., and Dean, J.R. (2016). Determination of carboxypeptidase activity in clinical pathogens by gas chromatography-mass spectrometry. Anal Lett 49(8), 1272-1277. doi: 10.1080/00032719.2015.1092153

Messner, K.R., and Imlay, J.A. (2002). Mechanism of superoxide and hydrogen peroxide formation by fumarate reductase, succinate dehydrogenase, and aspartate oxidase. $J$ Biol Chem 277(45), 42563-42571. doi: 10.1074/jbc.M204958200

Miller, J.L., Velmurugan, K., Cowan, M.J., and Briken, V. (2010). The type I NADH dehydrogenase of Mycobacterium tuberculosis counters phagosomal NOX2 activity to inhibit TNF-alpha-mediated host cell apoptosis. PLoS Pathog 6(4), e1000864. doi: 10.1371/journal.ppat.1000864.

Ngauy, V., Lemeshev, Y., Sadkowski, L., and Crawford, G. (2005). Cutaneous melioidosis in a man who was taken as a prisoner of war by the Japanese during World War II. J Clin Microbiol 43(2), 970-972.

Pumirat, P., Boonyuen, U., Vanaporn, M., Pinweha, P., Tandhavanant, S., Korbsrisate, S., and Chantratita, N. (2014). The role of short-chain dehydrogenase/oxidoreductase, induced 
by salt stress, on host interaction of B. pseudomallei. BMC Microbiol 14, 1. doi:

Pumirat, P., Cuccui, J., Stabler, R.A., Stevens, J.M., Muangsombut, V., Singsuksawat, E., Stevens, M.P., Wren, B.W., and Korbsrisate, S. (2010). Global transcriptional profiling of Burkholderia pseudomallei under salt stress reveals differential effects on the Bsa type III secretion system. BMC Microbiol 10, 171. doi: 10.1186/1471-2180-10-171

443 Pumirat, P., Vanaporn, M., Boonyuen, U., Indrawattana, N., Rungruengkitkun, A., and Chantratita, N. (2017). Effects of sodium chloride on heat resistance, oxidative susceptibility, motility, biofilm and plaque formation of Burkholderia pseudomallei. Microbiologyopen 6(4). doi: 10.1002/mbo3.493.

Ravishankar, S., Ambady, A., Swetha, R.G., Anbarasu, A., Ramaiah, S., and Sambandamurthy, V.K. (2016). Essentiality assessment of cysteinyl and lysyl-tRNA synthetases of Mycobacterium smegmatis. PLoS One 11(1), e0147188. doi: 10.1371/journal.pone.0147188

Ryan, A., Kaplan, E., Nebel, J.C., Polycarpou, E., Crescente, V., Lowe, E., Preston, G.M., and Sim, E. (2014). Identification of $\mathrm{NAD}(\mathrm{P}) \mathrm{H}$ quinone oxidoreductase activity in azoreductases from $P$. aeruginosa: azoreductases and $\mathrm{NAD}(\mathrm{P}) \mathrm{H}$ quinone oxidoreductases belong to the same FMN-dependent superfamily of enzymes. PLoS One 9(6), e98551. doi: 10.1371/journal.pone.0098551

Spero, M.A., Aylward, F.O., Currie, C.R., and Donohue, T.J. (2015). Phylogenomic analysis and predicted physiological role of the proton-translocating NADH:quinone oxidoreductase (complex I) across bacteria. MBio 6(2). doi: 10.1128/mBio.00389-15 
459 Stevens, J.M., Ulrich, R.L., Taylor, L.A., Wood, M.W., Deshazer, D., Stevens, M.P., and 460 Galyov, E.E. (2005). Actin-binding proteins from Burkholderia mallei and Burkholderia 461 thailandensis can functionally compensate for the actin-based motility defect of a 462 Burkholderia pseudomallei bimA mutant. J Bacteriol 187(22), 7857-7862. doi: 10.1128/JB.187.22.7857-7862.2005.

464 Weerakoon, D.R., Borden, N.J., Goodson, C.M., Grimes, J., and Olson, J.W. (2009). The role of 465 respiratory donor enzymes in Campylobacter jejuni host colonization and physiology. Microb Pathog 47(1), 8-15. doi: 10.1016/j.micpath.2009.04.009

467 Wiersinga, W.J., van der Poll, T., White, N.J., Day, N.P., and Peacock, S.J. (2006). Melioidosis: 468 insights into the pathogenicity of Burkholderia pseudomallei. Nat Rev Microbiol 4(4), 469 272-282. doi: 10.1038/nrmicro1385.

470 Wong, K.T., Puthucheary, S.D., and Vadivelu, J. (1995). The histopathology of human melioidosis. Histopathology 26(1), 51-55.

472 Wuthiekanun, V., Smith, M.D., Dance, D.A., and White, N.J. (1995). Isolation of Pseudomonas pseudomallei from soil in north-eastern Thailand. Trans $R$ Soc Trop Med Hyg 89(1), 4143. 


\section{Tables}

477 Table 1. Primers used in this study.

478 Table 2. Altered protein expression between $B$. pseudomallei wild-type K96243 and the 479 SDO mutant.

481 Figure legends

482

483

484 485

\section{Figure 1: Proteomic profiles of Burkholderia pseudomallei wild-type K96243 and the SDO} mutant

Representative 2-D gel of K96243 wild-type (A) and the SDO mutant (B). Quantitative intensity analysis revealed five differentially expressed protein spots between the two strains. Downregulated proteins are labeled with numbers. These differentially expressed proteins were subsequently identified by Q-TOF MS/MS (see Table 1).

Figure 2: Fold changes in gene expression of the kex, wzt, qor, proS and hsp genes in Burkholderia pseudomallei

RNA isolated from B. pseudomallei K96243, the SDO mutant, and the SDO complement cultured in $\mathrm{LB}$ broth at $37^{\circ} \mathrm{C}$ overnight was used for the determination of gene expression by quantitative real-time RT-PCR using the Brilliant II SYBR ${ }^{\circledR}$ Green QPCR Master Mix, one step (Agilent Technologies, Santa Clara, CA, USA) according to the manufacturer's recommendations. $23 S$ rDNA was used as a reference for the calculation of the relative expression levels of other genes. The normalized expression levels were calculated using the 
$4972^{-\Delta \Delta \mathrm{Ct}}$ method. Data represent the mean values, and error bars represent the standard deviations.

498 Asterisks indicate significant differences $(* \mathrm{P}<0.05)$ between strains.

499

500 Figure 3: Phenotypic examination of Burkholderia pseudomallei

501 (A) Adherence of B. pseudomallei to host cells. A549 cells were infected with overnight 502 cultures of B. pseudomallei K96243, the SDO mutant and the complemented strain at an MOI of 503 100. Adherent bacteria were counted after lysing infected cells at $3 \mathrm{~h}$ post-infection. (B) Biofilm

504 formation of $\boldsymbol{B}$. pseudomallei. A biofilm assay was performed for B. pseudomallei wild-type, 505 the SDO mutant and the complemented strains in LB broth using a 96-well plate. The degree of 506 biofilm formation was measured using a crystal violet stain at a wavelength of $630 \mathrm{~nm}$. (C) Heat 507 stress tolerance of $\boldsymbol{B}$. pseudomallei. The percent survival of $B$. pseudomallei after heat 508 treatment was investigated at $50^{\circ} \mathrm{C}$ for $15 \mathrm{~min} .100 \%$ viability corresponds to the colony forming 509 unit count of unexposed bacteria. The data were obtained from at least three experiments. Error

510 bars represent the standard deviation of the mean for experiments performed in triplicate.

511 Asterisks indicate significant differences $(* \mathrm{P}<0.05$ and $* \mathrm{P}<0.01)$ between groups.

512

\section{Supplemental Information}

514 Supplemental Information 1: Identification of spot 1 by mass spectrometry.

515 Supplemental Information 2: Identification of spot 2 by mass spectrometry.

516 Supplemental Information 3: Identification of spot 3 by mass spectrometry.

517 Supplemental Information 4: Identification of spot 4 by mass spectrometry.

518 Supplemental Information 5: Identification of spot 5 by mass spectrometry.

519 Supplemental Information 6: Growth kinetics of Burkholderia pseudomallei 
Figure 1

Proteomic profiles of Burkholderia pseudomallei wild-type K96243 and the SDO mutant

A

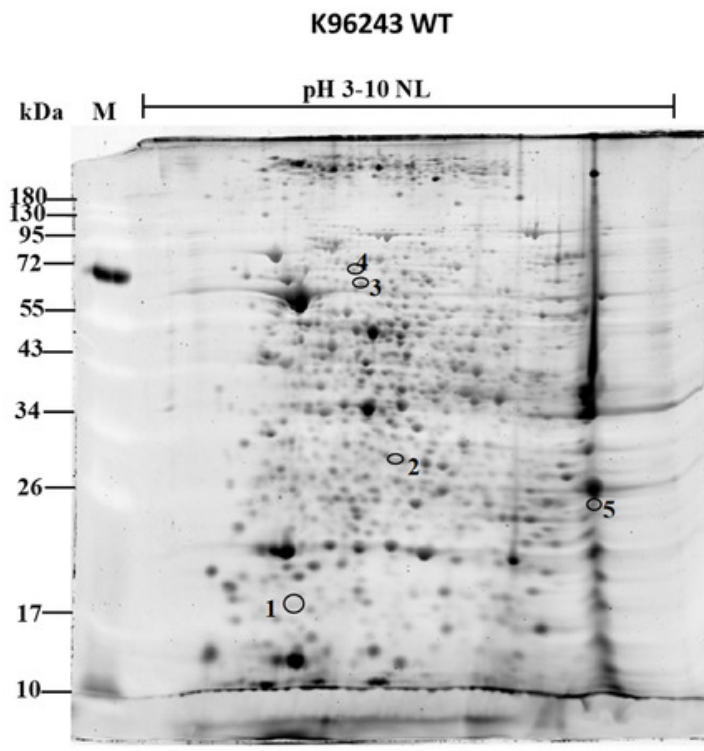

B

SDO mutant

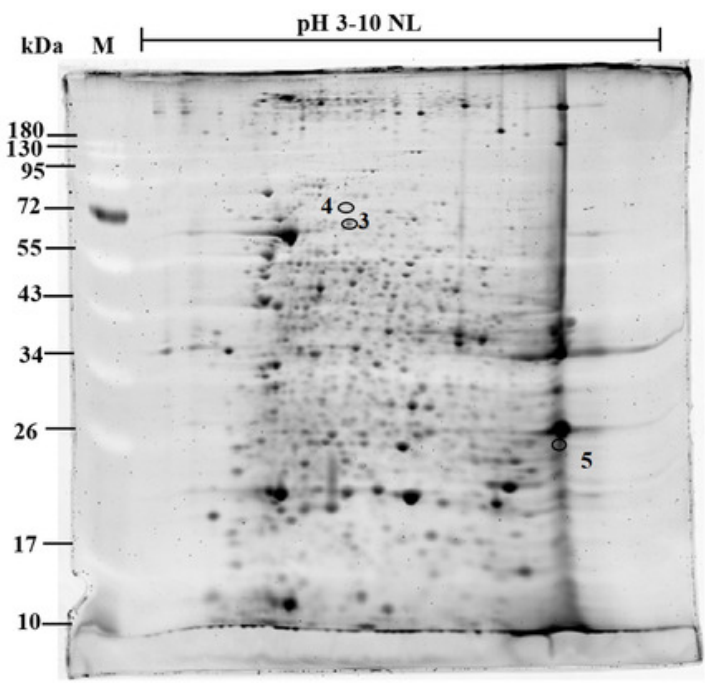


Figure 2

Fold changes in gene expression of the kex, wzt, qor, pros and hsp genes in Burkholderia pseudomallei

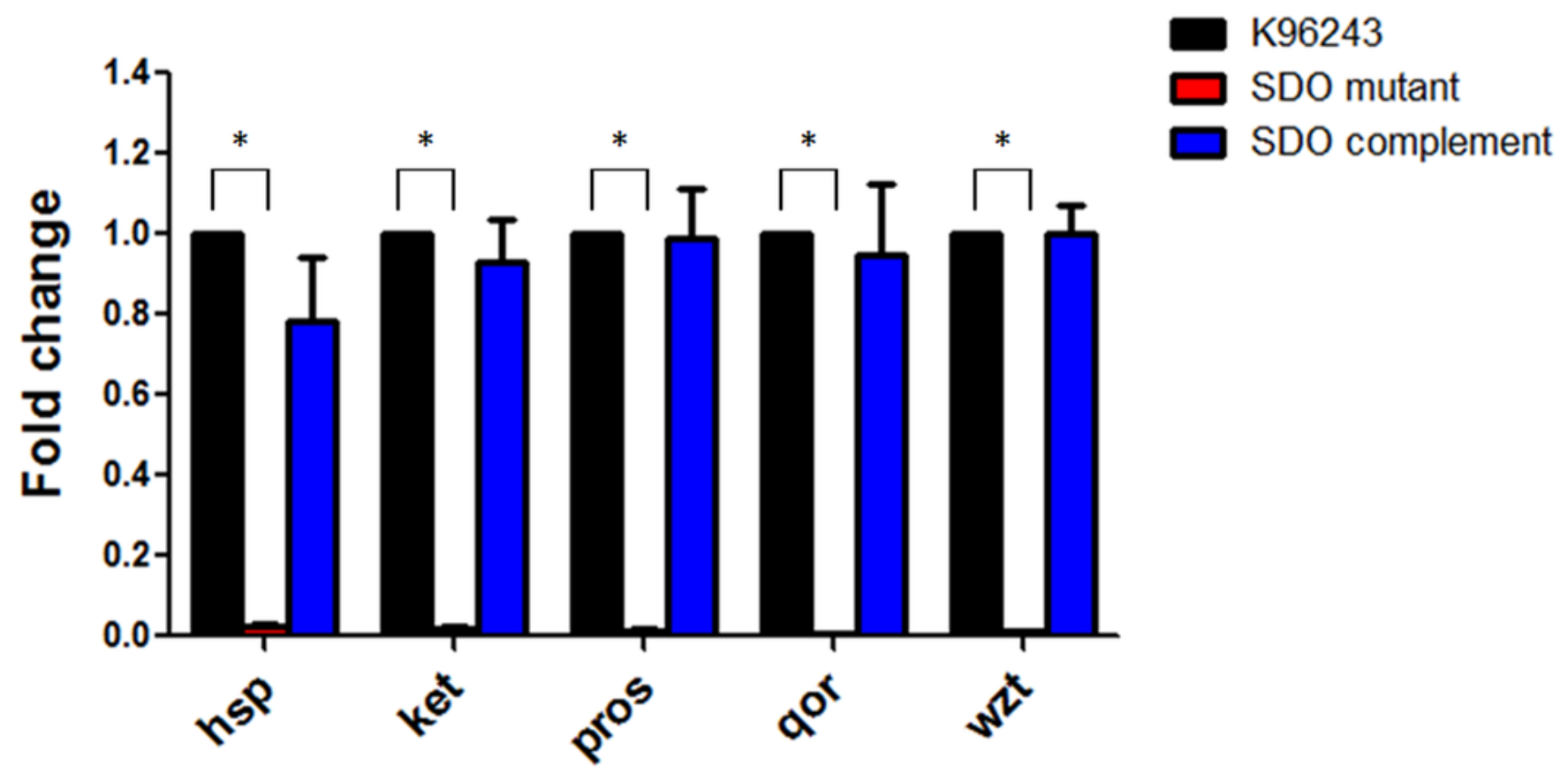


Figure 3

Phenotypic examination of Burkholderia pseudomallei 
A

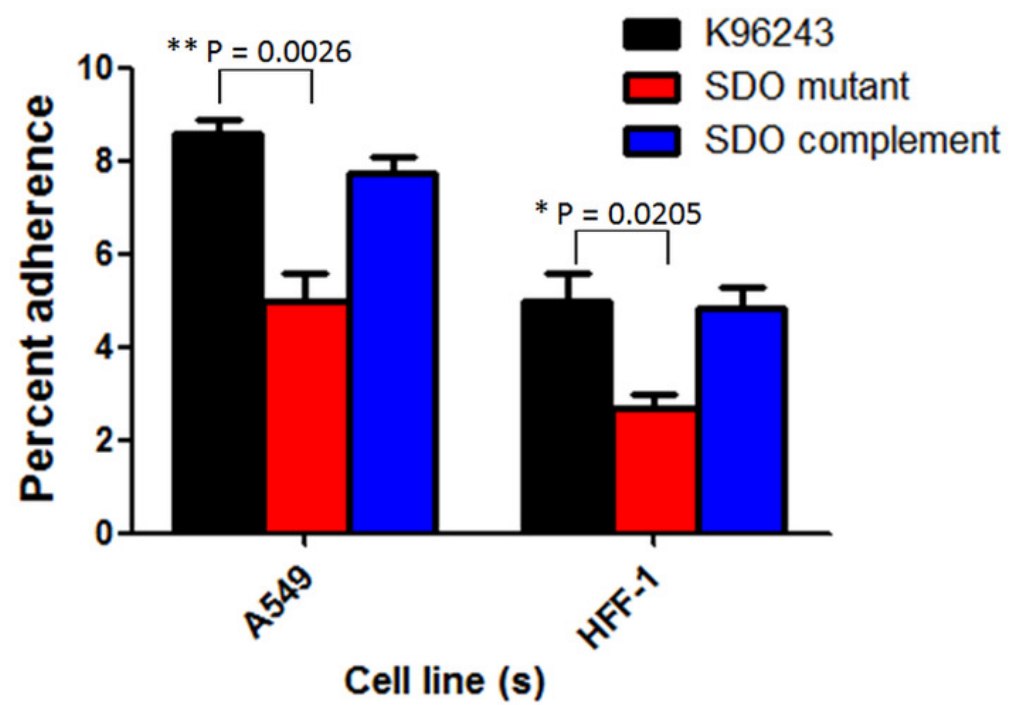

B

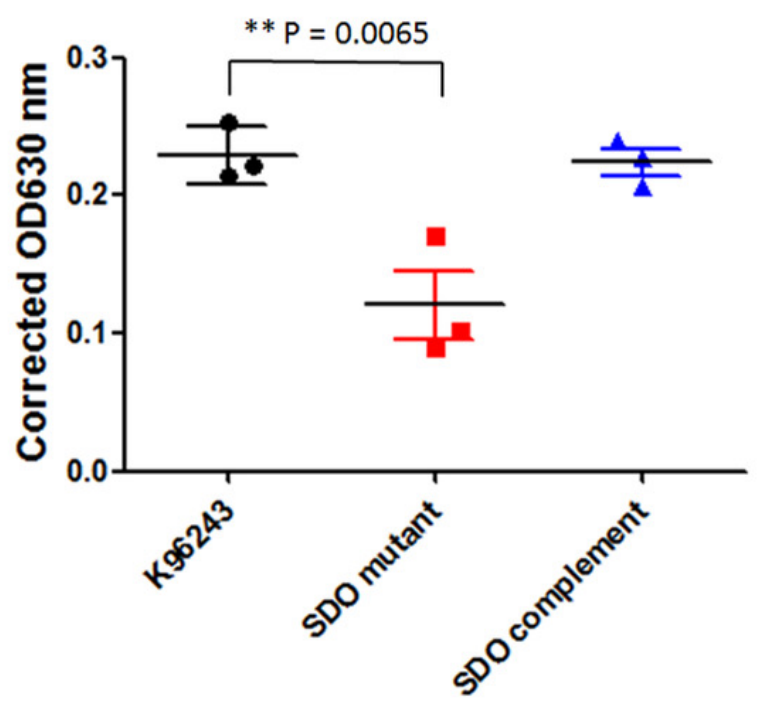

C

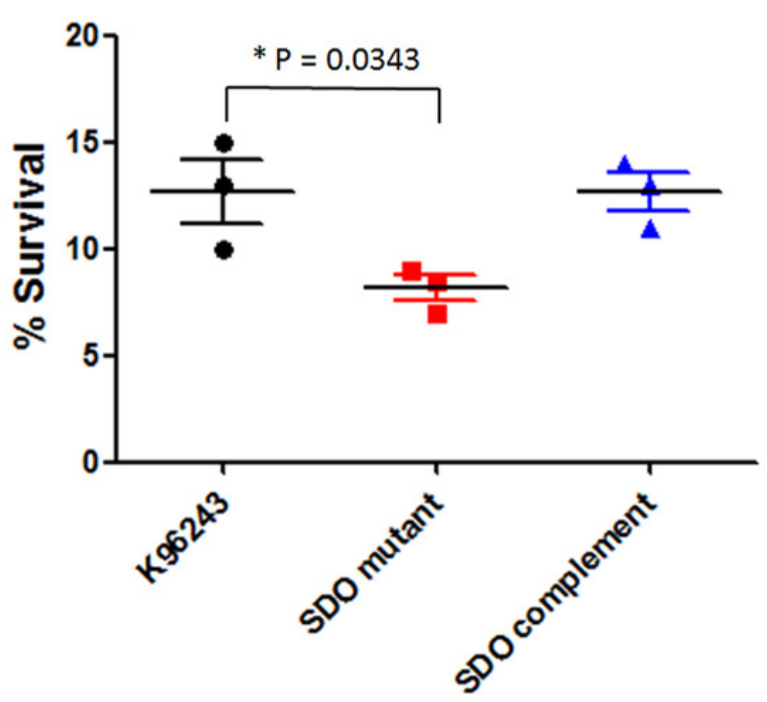


Table $\mathbf{1}$ (on next page)

Primers used in this study. 
1 Table 1. Primers used in this study

\begin{tabular}{|c|c|c|c|c|}
\hline $\begin{array}{l}\text { Primer } \\
\text { name }\end{array}$ & $\begin{array}{l}\text { Primer sequence } \\
\qquad\left(5^{\prime}-3^{\prime}\right)\end{array}$ & $\begin{array}{l}\text { Product } \\
\text { size (bp) }\end{array}$ & $\begin{array}{l}\text { Encoded } \\
\text { protein }\end{array}$ & Source \\
\hline Kex F & GTCGAGAATCGACGACTG & \multirow{2}{*}{150} & \multirow{2}{*}{$\begin{array}{c}\text { Putative } \\
\text { carboxypeptidase }\end{array}$} & \multirow{10}{*}{ This study } \\
\hline Kex R & CGCTATCTGACGAAGCAC & & & \\
\hline Wzt F & GACGACCTGCTGATTCTG & & Glutamate/aspartate & \\
\hline Wzt R & CCAAGGAGATGACAACGA & & $\mathrm{ABC}$ transporter & \\
\hline Qor F & CACGTCCGCTTACCTGAT & \multirow{2}{*}{154} & \multirow{2}{*}{ Quinone oxidoreductase } & \\
\hline Qor R & CTTCTCGTCGCTCGACAC & & & \\
\hline ProS F & AGATGCCGGTGAACTTCT & \multirow{2}{*}{165} & \multirow{2}{*}{ Prolyl-tRNA synthetase } & \\
\hline ProS R & CGTACGCGTCGTACATCT & & & \\
\hline Hsp F & GGCGAACATATTCTGCTG & \multirow{2}{*}{176} & \multirow{2}{*}{ Hsp70 family protein } & \\
\hline Hsp R & GGAACTGCTTGTGCTGAC & & & \\
\hline $23 \mathrm{~s} \mathrm{~F}$ & TTTCCCGCTTAGATGCTTT & \multirow{2}{*}{343} & \multirow{2}{*}{ 23S RNA } & (Pumirat et al., \\
\hline $23 \mathrm{~s} \mathrm{R}$ & AAAGGTACTCTGGGGATAA & & & 2010) \\
\hline
\end{tabular}

2 


\section{Table 2 (on next page)}

Altered protein expression between B. pseudomallei wild-type K96243 and the SDO mutant. 
1 Table 2. Altered protein expression between B. pseudomallei wild-type K96243 and the SDO mutant

\begin{tabular}{|c|c|c|c|c|c|c|c|c|c|}
\hline Protein & Accession no. & M.W. & pI & \%Cov & No. of peptide & K96243 & SDO & Ratio & ANOVA \\
\hline Putative carboxypeptidase & CDU31600 & 60112 & 5.5 & 43.9 & 19 & 0.31 & 0.05 & 0.15 & 0.009 \\
\hline Glutamate/aspartate $\mathrm{ABC}$ transporter & ABN82061 & 36306 & 7.9 & 48.8 & 15 & 0.15 & 0.02 & 0.14 & 0.045 \\
\hline Prolyl-tRNA synthetase & SYP_BURMA & 63413 & 5.5 & 28.4 & 14 & 0.19 & 0.04 & 0.23 & 0.035 \\
\hline Hsp70 family protein & WP_004524146 & 65225 & 5.7 & 13.8 & 5 & 0.1 & 0 & 0 & 0.006 \\
\hline Quinone oxidoreductase & WP_027716432 & 35439 & 5.5 & 2.4 & 1 & 0.06 & 0 & 0 & 0.001 \\
\hline
\end{tabular}

